EXTENDED REPORT

\title{
Detection of small joint synovitis by ultrasonography: the learning curve of rheumatologists
}

\author{
M-A D'Agostino, J-F Maillefert, R Said-Nahal, M Breban, P Ravaud, M Dougados
}

Ann Rheum Dis 2004;63:1284-1287. doi: 10.1136/ard.2003.012393

See end of article for authors' affiliations

Correspondence to: Professor Maxime Dougados, Service de Rhumatologie B, Hôpital Cochin, 27 rue du Faubourg Saint Jacques, 75014, Paris, France; maxime.dougados@ cch.ap-hop-paris.fr

Accepted

19 November 2003
Background: Ultrasonography allows assessment of soft tissue structures and has become a valued tool for diagnosing synovitis.

Objective: To assess the learning curve for ultrasonography in evaluating synovitis of the small joints in rheumatoid arthritis.

Methods: Metacarpophalangeal (MCP), metatarsophalangeal (MTP), and proximal interphalangeal (PIP) joints were evaluated using ultrasonography (Esaote AU 5 Epi, linear probe 10-13 MHz) by four rheumatologists, the first being experienced (senior), the others having no (fellows 1 and 2) or little (fellow 3) experience in ultrasonography. For each fellow, the learning curve was divided into blocks. In each block the fellow examined five consecutive patients with the senior; then, blinded to the senior's results, two further patients alone (seven patients examined per block). For each evaluation, the MCP, PIP, and MTP joints were individually tagged as having synovitis or not. The ultrasonography results were compared between fellow and senior for the two last patients of each block, using proportions of agreement and $\kappa$ statistics.

Results: 70 patients were evaluated (seven practice patients, followed by nine blocks). For fellows 1 and 2, the proportions of agreement were respectively $42 \%$ and $47 \%$ ( $\kappa=0$ and 0 ) at the first evaluation, and rose progressively to $82 \%$ and $82 \%(\kappa=0.63$ and 0.62$)$ at the ninth evaluation. For fellow 3 , initially good results were followed by decreased accuracy.

Conclusions: Detecting synovitis of the MCP, PIP, and MTP joints using ultrasonography can be done accurately by rheumatologists initially not experienced in this technique. At least 70 examinations were necessary to develop competence.
$\mathrm{R}$ heumatoid arthritis is characterised by synovitis of the metacarpophalangeal (MCP), metatarsophalangeal (MTP), and proximal interphalangeal (PIP) joints. The assessment of patients with rheumatoid arthritis, in both clinical practice and research, includes evaluation of the MCP joints, the finger PIP joints, and, mostly in clinical practice, the MTP joints. Synovitis of these joints is an important aspect of the diagnosis of this disease. A diagnosis of rheumatoid arthritis can be made subjectively by a rheumatologist on the basis of experience, or in a more standardised manner using the American College of Rheumatology (ACR) criteria, ${ }^{1}$ which include synovitis of the small joints. However, both methods are of limited value for predicting whether rheumatoid arthritis is present in patients with early arthritis. Subjective diagnosis has good sensitivity but poor specificity, while the ACR criteria have better specificity but limited sensitivity. ${ }^{2}$ Thus, although there is strong evidence that patients with rheumatoid arthritis should be treated as early as possible, ${ }^{3}$ we lack an accurate tool for diagnosing the disease at an early stage.

Because the clinical assessment of small joint synovitis can be difficult, ${ }^{4}$ techniques that increase our ability to detect synovitis in these joints could be useful in both clinical practice and research, by improving the diagnosis of rheumatoid arthritis and the assessment of established disease. Ultrasonography allows the assessment of soft tissue structures and has become a valued tool for diagnosing synovitis. ${ }^{56}$ This technique is therefore potentially important in the management of rheumatoid arthritis, both for diagnosis and for disease monitoring and the assessment of treatment efficacy. ${ }^{6-8}$ It might also become an important outcome measure in therapeutic trials in rheumatoid arthritis. However, ultrasonography is an operator dependent imaging tool. The use of ultrasonography in clinical practice implies that practitioners involved in the diagnosis and management of rheumatoid arthritis (rheumatologists) should detect synovitis accurately in their patients, especially in the small joints.

Most rheumatologists do not have adequate training in ultrasonography. Also, "adequate training" has yet to be defined. The development of ultrasonographic detection of synovitis in small joints depends on the time needed to acquire the necessary skills, particularly for busy outpatient rheumatologists. Our aim in this study was to assess the rate at which rheumatologists with little or no experience in ultrasonography develop adequate skills to undertake an ultrasonographic evaluation for synovitis in the small joints in patients with rheumatoid arthritis.

\section{METHODS}

MCP, finger PIP, and MTP joints of consecutive hospital inpatients with rheumatoid arthritis (ACR criteria) were evaluated. The ultrasonography equipment was an Esaote AU 5 Epi (Genoa, Italy) with a $10-13 \mathrm{MHz}$ linear assay transducer. The evaluation was done by four rheumatologists: the senior, who was experienced in musculoskeletal ultrasonography; fellows 1 and 2, who had no experience in ultrasonography; and fellow 3, who had a little experience in ultrasonography, but not in the evaluation of synovitis, and who had not been practising for several years. The fellows' professional situations were somewhat different: fellow 1 was a 38 year old rheumatologist experienced in clinical practice and research, who was completing a few months' period of clinical research without visiting patients; fellow 2 was a 33 year old rheumatologist who had been working in a clinical research unit for several years; fellow 3 was a 38 year 
old experienced rheumatologist who was working both as a researcher and as a clinical practitioner.

\section{Study design}

The study was divided into two parts: the training session, followed by the learning curve.

\section{Training session}

At the beginning of the study, the fellows underwent five hours of "hands-on" training and didactic instructions focused on the ultrasonographic examination of small joints. At the end of this session seven training patients were examined by fellows: the ultrasonographic examination of the first five patients was supervised by the senior, and the last two patients were evaluated by the senior and by the fellows in the presence of the senior. In the latter case the senior did not help the fellows to interpret the examination but helped them to perform the examination correctly.

\section{Learning curve session}

The learning curve was divided into seven-patient blocks: five consecutive patients examined with the senior-that is, practice patients-followed by two patients examined without assistance-evaluation patients. The senior carried out the examination of the practice patients, while the fellows watched the monitor and decided whether the joints showed synovitis or not. The senior then corrected and commented the fellows' responses. Next, the two other patients were examined separately by the fellows and the senior, the fellows being blinded to the senior's evaluation results. This was done in order to avoid multiple examinations per patient, for ethical reasons. For each evaluation, the MCP, PIP, and MTP joints were individually tagged as having synovitis or not. Each patient was examined by fellows and the senior on the same day.

\section{Ultrasonography}

Each joint was scanned in the longitudinal plane on the dorsal aspect with the hand in a neutral position. This scan was preferred to a palmar or plantar scan because of its reliability for detecting synovitis. The MCP, finger PIP, and MTP joints were scanned consecutively from the first to the fifth joint. The joints were examined for capsular distension and synovial proliferation (indicating synovitis) (fig 1). We considered a joint to be normal when the capsule stretched linearly between the metacarpal head and the phalangeal base, and the only anechoic area present in the joint was the cartilage line. Synovitis was identified as a hypoechoic area (inflammatory synovitis) or a hyperechoic area (fibrous synovitis) in the joint space. Synovial effusion was defined as an anechoic area. The presence of a well defined area of increased echogenicity within the extensor tendon sheath was considered as synovial thickening if it was detected in front of the neck of metacarpal head. ${ }^{7}$ At the end of each joint evaluation, the sonographer noted subjectively whether or not the joint had synovitis.

\section{Statistical analysis}

Statistical analysis was carried out on the results of the examination of the last two practice patients and the two evaluation patients. For each pair of patients, the fellows' results were compared with the gold standard-that is, the senior's results-using proportions of agreement and $\kappa$ statistics $(0=$ no synovitis, $1=$ synovitis $) .{ }^{9}$ The fellows subjectively noted that the evaluation of finger PIP was more difficult than MCP or MTP. We therefore undertook two analyses: one included every evaluated joint (60 joints, 30 per patient), the other only MCP and MTP joints (40
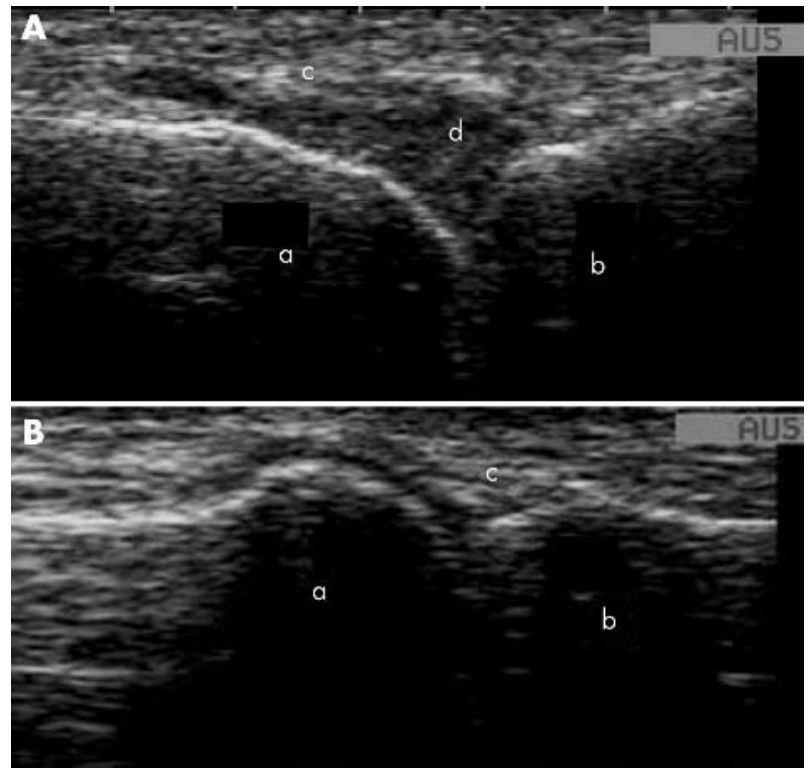

Figure 1 Ultrasonographic picture of second metatarsophalangeal joint with synovitis (panel A) and without synovitis (panel B). a, metatarsal head; $b$, base of phalanges; $c$, joint capsule (side view); $d$, synovitis; e, joint effusion.

joints). Bearing in mind that many outcome measures for rheumatoid arthritis do not include the MTP joints, we conducted a third analysis on MCP and PIP joints alone.

Linear regression analysis was used to evaluate the relation between the number of joints considered by the senior as having synovitis and the $\kappa$ coefficients. Sensitivity (percentage of fellow-tagged synovitis joints among the seniortagged synovitis joints), and specificity (percentage of fellow-tagged non-synovitis joints among the senior-tagged non-synovitis joints) were evaluated at the end of the training session, and at the first and last evaluation.

\section{RESULTS}

In all, 70 patients including seven training patients, were evaluated by each fellow (except for fellow 3, see below). According to the protocol, 20 of these patients were actively evaluated by fellows alone, the remainder being evaluated passively, and not hands-on. The total number of joints examined was 2100 (210 for the training session and 1890 for the learning curve). The fellows scanned 60 joints hands-on during the training session and 360 joints during the learning curve session. The patients were examined during a continuous three month period.

The overall mean (SD) numbers of joints with synovitis in the evaluated patients (examined separately by the fellows) were: 24.4 (9) and 27 (5) (fellow 1 and senior, respectively); 26.2 (7) and 29.8 (7) (fellow 2 and senior); 26.9 (4) and 30.4 (4) (fellow 3 and senior).

At the end of the training period, the proportions of agreement were $60 \%$ and $85 \%(\kappa=0.22$ and 0.7$)$ for fellow $\mathrm{l}$ and 2 , respectively. The fellows' sensitivity and specificity for synovitis were $77.8 \%$ and $45.5 \%$, respectively (fellow 1 ), and $81.5 \%$ and $87.9 \%$, respectively (fellow 2). However, these initially good results were not confirmed and probably reflected the presence of the senior during the evaluation. At the first evaluation of the learning curve, the proportions of agreement were $42 \%$ and $47 \%(\kappa=0$ and 0$)$ for fellows 1 and 2, respectively, and rose progressively to $82 \%$ and $82 \%$ $(\kappa=0.63$ and 0.62$)$ at the ninth evaluation (fig $2 \mathrm{~A}$ and $2 \mathrm{~B}$ ). At the first evaluation of the learning curve, the sensitivity and specificity of the examination of fellow 1 were $11.5 \%$ and 

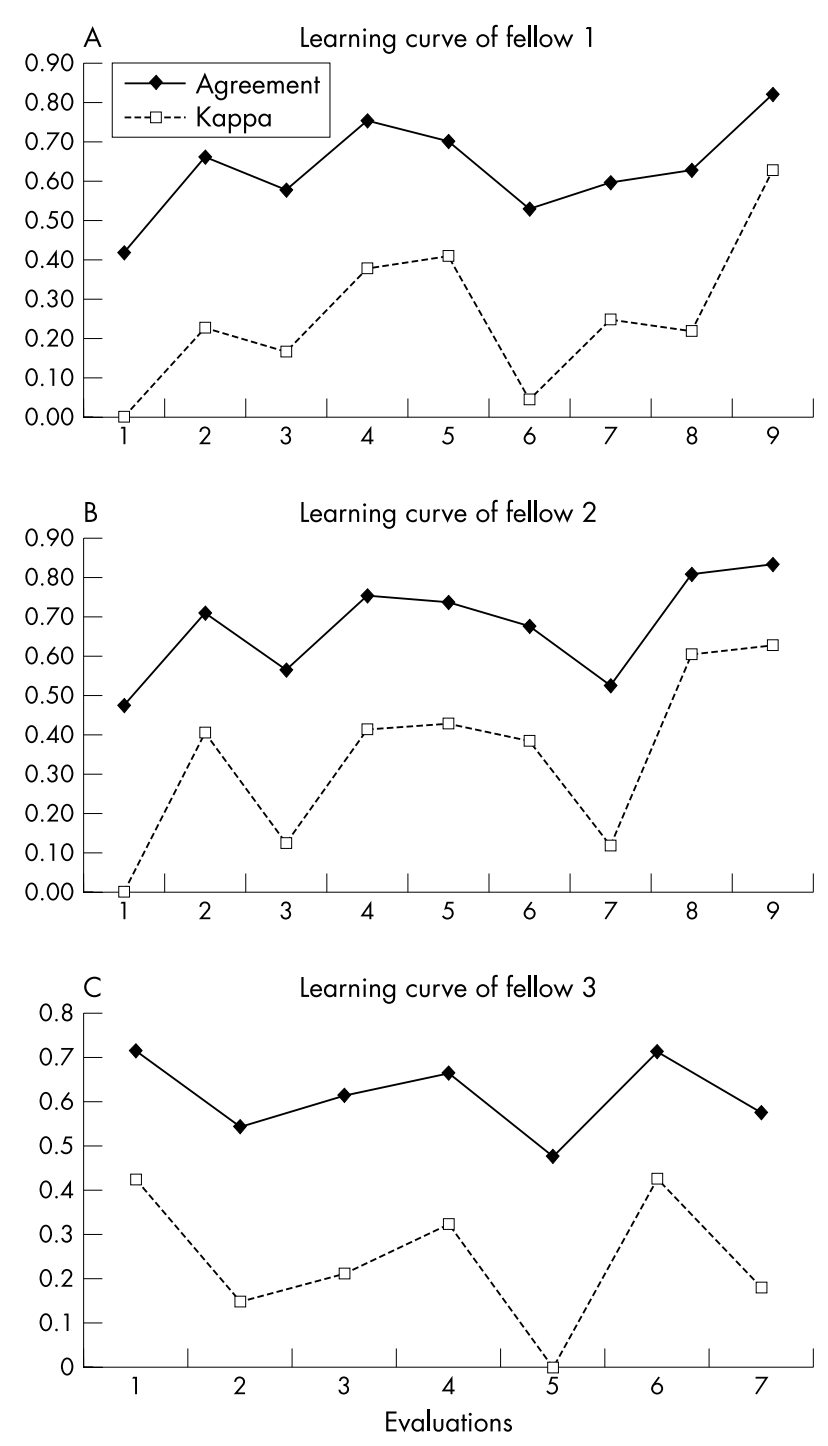

Figure 2 Non-experienced rheumatologists' learning curve for detecting synovitis of the metacarpophalangeal (MCP),

metatarsophalangeal (MTP), and proximal interphalangeal (PIP) joints using ultrasonography in patients with rheumatoid arthritis. Proportions of agreement and $\mathrm{K}$ coefficients from the first to the ninth evaluation for fellows $1(A)$ and $2(B)$, and from the first to the seventh evaluation for fellow 3 (C)

$64.7 \%$ respectively, reaching $80.6 \%$ and $82.7 \%$ at the ninth evaluation. The sensitivity and specificity of fellow 2 were $35 \%$ and $52.5 \%$ at the first evaluation, and reached $81.1 \%$ and $82.6 \%$ at the ninth evaluation.

Fellow 3 evaluated only 56 patients (seven practice patients, plus seven blocks of seven evaluation patients). His learning curve was different, with good results at the start and a subsequent decrease in $\kappa$ coefficients and proportions of agreement (fig 2C). The proportions of agreements and the $\kappa$ coefficients were $80 \%$ and 0.6 , respectively, at the end of the training session, $72 \%$ and 0.43 at the first evaluation, and $58 \%$ and 0.18 at the seventh evaluation. At the end of the training session, sensitivity and specificity for synovitis were $80.6 \%$ and $79.3 \%$, respectively, then $71 \%$ and $72.4 \%$ at the first evaluation, decreasing to $52.8 \%$ and $66.7 \%$, respectively at the seventh evaluation.

There was no relation between the number of seniortagged cases of synovitis and the $\kappa$ coefficients $(p=0.88$, $r=-0.12 ; \mathrm{p}=0.89$ and $r=-0.12 ; \mathrm{p}=0.7$ and $r=-0.13$, for fellows 1,2 , and 3, respectively).
Similar results were found for the additional analyses: MCP and MTP, or MCP and PIP (data not shown).

\section{DISCUSSION}

In our study, 70 examinations were necessary (including the training session) for rheumatologists not experienced in ultrasonography to assess synovitis accurately in the small joints in patients with rheumatoid arthritis. This figure of 70 examinations may be considered excessive and to require too much time out of the schedule of a busy rheumatologist. However, it is comparable with figures found in other studies evaluating learning curves for other ultrasonographic investigations. ${ }^{10}{ }^{11}$ In a survey completed at the first BSR Course in Musculoskeletal Ultrasound for Rheumatologists, delegates expected to dedicate a mean of 2.1 hours a week to carrying out ultrasonography. ${ }^{12}$ In our study, the time spent by the fellows in learning the skills was not measured. The mean number of patients examined a week was just above 5, and each examination lasted from 20 to 30 minutes, which yields a mean time of about 2.1 hours a week. However, the results differed from one fellow to another. At the end of the training session, the agreement between the senior and fellow 3 was very good, and could be considered as a standard for a first evaluation in comparison with the other fellows.

At the first evaluation, the accuracy of fellows 1 and 2 was very poor in comparison with the training session. However, their accuracy improved from then onwards until the ninth evaluation. As the curves of proportions of agreement and $\kappa$ coefficients did not reach a plateau, one could suggest that the study was interrupted too soon and that the accuracy would have improved if the process had been extended. That might be true, but the sensitivity and specificity for synovitis, as well as the proportions of agreements, reached over $80 \%$, which can be considered good. Additionally, a $\kappa$ value between 0.6 and 0.8 is considered good. ${ }^{9}$ One can argue that our course of instruction could be improved: of the 70 evaluated patients, only 20 were actively evaluated by the fellows alone, the remainder being evaluated passively, and not hands-on. Competence may be acquired faster if the evaluation is more active.

The results of fellow 3 are very interesting and should be discussed separately. Contrary to the other fellows, fellow 3 had some experience with ultrasonography, which might explain the good results at the outset. However, he had not been practising for several years and had no previous experience of ultrasonographic assessment of synovitis. Contrary to the other fellows, fellow 3 showed a decrease over time in his accuracy in detecting synovitis. It should be noted that fellows 1 and 2 had spare time for training in ultrasonography, but fellow 3 often had to interrupt patient visits for his ultrasonographic training, which he had to rush. He eventually became less and less enthusiastic about the training. This suggests that in order to obtain proper training in ultrasonography, a rheumatologist must display enthusiasm and afford enough spare time to acquire the skills.

We can therefore state that with our training protocol, 70 examinations led to good accuracy in detecting synovitis of the small joints by ultrasonography, although better accuracy might be acquired with further training.

These results show that if properly trained, rheumatologists can detect synovitis accurately in the small joints of patients with rheumatoid arthritis using ultrasonography. Ultrasonography may eventually be required for medical specialists' training in rheumatology (as is presently the case in several countries). Also, the postgraduate training of rheumatologists, who agree to spend sufficient time and are supported by colleagues in radiology, could lead to the use of ultrasonography in clinical practice for diagnostic purposes and for the management of rheumatoid arthritis. 
Furthermore, our results suggest that acquiring the necessary skills would not represent a major difficulty if investigators wished to supplement multicentre therapeutic trials with ultrasonographic evaluation for synovitis.

\section{Authors' affiliations}

M-A D'Agostino, R Said-Nahal, M Breban, M Dougados, René Descartes University, Rheumatology B Division, AP-HP, Cochin Hospital, Paris, France

J-F Maillefert, Dijon University, Rheumatology Department and INSERM/ERIT-M 0207, Dijon, France

P Ravaud, Department of Epidemiology, AP-HP, Bichat Hospital, Paris, France

\section{REFERENCES}

1 Arnett FC, Edworthy SM, Bloch DA, McShane DJ, Fries JF, Cooper NS, et al. The American Rheumatism Association 1987 revised criteria for the classification of rheumatoid arthritis. Arthritis Rheum 1988:31:315-24.

2 Saraux A, Berthelot JM, Chalès G, Le Henaff C, Thorel JB, Hoang S, et al. Ability of the American College of Rheumatology 1987 criteria to predict rheumatoid arthritis in patients with early arthritis and classification of these patients two years later. Arthritis Rheum 2002;44:2485-91.
3 Emery P, Breedveld FC, Dougados M, Kalden JR, Schiff MH, Smolen JS. Early recommendation for newly diagnosed rheumatoid arthritis: evidence based development of a clinical quide. Ann Rheum Dis 2002:61:290-7.

4 Goupille P, Roulot B, Akoka S, Avimadje AM, Garaud P, Naccache L, et al. Magnetic resonance imaging: a valuable method for the detection of synovial inflammation in rheumatoid arthritis. J Rheumatol 2001;28:35-40.

5 Conaghan PG, McGonagle D, Wakefield R, Emery P. New approaches to imaging of early rheumatoid arthritis. Clin Exp Rheumatol 1999; 17(suppl 18):S37-42.

6 Backhaus M, Kamradt T, Sandrock D, Loreck D, Fritz J, Wolf KJ, et al. Arthritis of the finger joints. A comprehensive approach comparing conventional radiography, scintigraphy, ultrasound, and contrast enhanced magnetic resonance imaging. Arthritis Rheum 1999;42:1232-45.

7 Grassi W, Lamanna G, Farina A, Cervini C. Synovitis of small joints: sonographic guided diagnosis and therapeutic approach. Ann Rheum Dis 1999;58:595-7.

8 Stone M, Bergin D, Whelan B, Maher M, Murray J, McCarthy C. Power Doppler ultrasound assessment of rheumatoid hand synovitis. J Rheumatol 2001;28:1979-82.

9 Armitage P, Berry G. Statistical methods in medical research, 3rd ed. Oxford: Blackwell Scientific Publications, 1994

10 Rozycki GS, Ballard RB, Feliciano DV, Schmidt JA, Pennington SC. Surgeonperformed ultrasound for the assessment of truncal injuries: lessons learned from 1540 patients. Ann Surg 1998;228:557-67.

11 Röthlin MA, Näf R, Amgwerd M, Candinas D, Frick T, Trentz O. Ultrasound in blunt abdominal and thoracic trauma. J Trauma 1993;34:488-95.

12 Speed CA, Bearcroft PWP. Training in musculoskeletal sonography: report from the first BSR course. Rheumatology 2002;41:346. 